\title{
G. Czédli's tolerance factor lattice construction and weak ordered relations
}

\author{
Sándor Radeleczki
}

\begin{abstract}
G. Czédli proved that the blocks of any compatible tolerance $T$ of a lattice $L$ can be ordered in such a way that they form a lattice $L / T$ called the factor lattice of $L$ modulo $T$. Here we show that the DedekindMacNeille completion of the lattice $L / T$ is isomorphic to the concept lattice of the context $(L, L, R)$, where $R$ stands for the reflexive weak ordered relation $\leq \circ T$. Weak ordered relations constitute the generalization of the ordered relations introduced by S. Valentini. Reflexive weak ordered relations can be characterized as compatible reflexive relations $R \subseteq L^{2}$ satisfying $R=\leq \circ R \circ \leq$.
\end{abstract}

Mathematics Subject Classification. 06B15, 06B23, 06A15, 06 B05.

Keywords. Compatible tolerance, Dedekind-MacNeille completion of a lattice, Weak ordered relation, Formal context, Concept lattice.

\section{Introduction}

A binary relation on a (complete) lattice $L$ is called (completely) compatible if it is a (complete) sublattice of the direct product $L^{2}=L \times L$. A reflexive symmetric and (completely) compatible relation $T \subseteq L^{2}$ is a called a (complete) tolerance of $L$. All tolerances of a lattice $L$, denoted by $\operatorname{Tol}(L)$ form an algebraic lattice (with respect to the inclusion).

Let $T \in \operatorname{Tol}(L)$ and $X \subseteq L, X \neq \emptyset$. If $X^{2} \subseteq T$, then the set $X$ is called a preblock of $T$. Blocks are maximal preblocks (with respect to $\subseteq$ ). It is known that the blocks of any tolerance $T$ are convex sublattices of $L$. In [1] G. Czédli proved that the blocks of $T$ can be ordered in such a way that they form a lattice. This lattice is denoted by $L / T$ and it is called the factor lattice of

Presented by R. Pöschel.

This research started as part of the TAMOP-4.2.1.B-10/2/KONV-2010-0001 project, supported by the European Union, co-financed by the European Social Fund 113/173/0-2. 
$L$ modulo $T$. The notion of factor lattice constructed with his method is a natural generalization of factor lattice by a congruence.

Definition 1.1. We say that a binary relation $R$ is a weak ordered relation on the lattice $L$ if it satisfies the following conditions:

(1) for any $u, x, y, z \in L, u \leq x,(x, y) \in R$ and $y \leq z$ imply $(u, z) \in R$;

(2) given any $t \in L$ and any nonempty finite $A \subseteq L$, if $(a, t) \in R$ holds for each $a \in A$ then $(\bigvee A, t) \in R$;

(3) given any $z \in L$ and any nonempty finite $A \subseteq L$, if $(z, a) \in R$ holds for each $a \in A$ then $(z, \bigwedge A) \in R$.

Clearly, if conditions (2) and (3) hold for any two-element subset of $L$, then they hold for arbitrary finite and nonempty $A \subseteq L$.

An ordered relation $R$ on a complete lattice $L$ is a weak ordered relation which satisfies conditions (2) and (3) for arbitrary (i.e. even infinite or empty) $A \subseteq L$. This notion was introduced by S. Valentini [8], and in [5] it was shown that any ordered relation is a completely compatible relation on $L$. We will prove that reflexive weak ordered relations of $L$ can be characterized as compatible reflexive relations $R \subseteq L^{2}$ satisfying $R=\leq \circ R \circ \leq$. Moreover, we will see that for any $T \in \operatorname{Tol}(L), \leq \circ T=\leq \circ T \circ \leq$ and $R:=\leq \circ T$ is a weak ordered relation with the property that $T=R \cap R^{-1}$, where $R^{-1}$ stands for the inverse relation of $R$. The set of weak ordered relations and that of reflexive weak ordered relations of a lattice $L$ will be denoted by $\operatorname{WOR}(L)$ and $\operatorname{ReWOR}(L)$, respectively.

The main results of the paper point out the connection between weak ordered relations and factor lattices defined by tolerances. It is proved that for any tolerance $T$ of a lattice $L$ the Dedekind-MacNeille completion of $L / T$ is isomorphic to the concept lattice $\mathcal{L}(L, L, R)$ of the context $(L, L, R)$, where $R:=\leq \circ T$. It is also shown that the blocks of $T$ correspond exactly to the concepts $(A, B) \in \mathcal{L}(L, L, R)$ having the property that $A \cap B \neq \emptyset$. This result generalizes a result of [6], where for any complete lattice $L$ and any complete tolerance $T \subseteq L^{2}$ the isomorphism $L / T \cong \mathcal{L}(L, L, \leq \circ T)$ was established.

The paper is structured as follows: In Section 2 some basic notions and the interrelation between the lattices $\operatorname{Tol}(L)$ and $\operatorname{ReWOR}(L)$ are presented. In Section 3 , the concept lattice $\mathcal{L}(L, L, \leq \circ T)$ is described and the main results of the paper are presented.

\section{Reflexive weak ordered relations and FCA notions}

First, observe that weak ordered relations on a lattice $L$ are also compatible relations. Indeed, let $R \in \operatorname{WOR}(L)$ and $\left(x_{1}, y_{1}\right),\left(x_{2}, y_{2}\right) \in R$. Condition (1) of Definition 1.1 implies $\left(x_{1}, y_{1} \vee y_{2}\right),\left(x_{2}, y_{1} \vee y_{2}\right) \in R$ and $\left(x_{1} \wedge x_{2}, y_{1}\right),\left(x_{1} \wedge\right.$ $\left.x_{2}, y_{2}\right) \in R$. Now, by using conditions (2) and (3) we obtain $\left(x_{1} \vee x_{2}, y_{1} \vee y_{2}\right) \in R$ and $\left(x_{1} \wedge x_{2}, y_{1} \wedge y_{2}\right) \in R$, proving the compatibility of $R$.

The relations $\leq$ and $\nabla=L \times L$ are examples of reflexive ordered relations. We consider the empty relation $\emptyset$ also as a weak ordered relation. Clearly, 
relation $\emptyset$ is not reflexive. Another weak ordered relation which is not reflexive in general, is given in the following

Example 2.1. Let $f: L \rightarrow L$ be a join-endomorphism of the lattice $L$. Then the binary relation

$$
R^{f}:=\left\{(x, y) \in L^{2} \mid f(x) \leq y\right\}
$$

is a weak ordered relation on the lattice $L$.

We note that the above relation appears already in Valentini's paper [8] who proved that in case of a complete join-endomorphism $R^{f}$ is an ordered relation. Similarly, the "complete versions" of the claims listed in the next proposition appear in [8] with almost the same proofs.

Proposition 2.2. Let $L$ be a lattice. Then $(\operatorname{WOR}(L), \subseteq)$ is a complete lattice, and $(\operatorname{WOR}(L), \circ)$ is a monoid with unit element $\leq$. In addition, the relations

$$
\begin{aligned}
& \left(R_{1} \cap R_{2}\right) \circ S=\left(R_{1} \circ S\right) \cap\left(R_{2} \circ S\right) \\
& S \circ\left(R_{1} \cap R_{2}\right)=\left(S \circ R_{1}\right) \cap\left(S \circ R_{2}\right)
\end{aligned}
$$

hold for any $R_{1}, R_{2}, S \in \operatorname{WOR}(L)$, that is, o distributes over intersections from both sides.

Proof. Since The exprssion Wor $(L)$ has to be formatted everywhere in the paper as in Proposition 2.2 Wor $(L)$ is closed with respect to arbitrary intersections, including the empty intersection, it follows that $(\operatorname{WOR}(L), \subseteq)$ is a complete lattice. Let $R, S \in \mathrm{WOR}(L)$. We prove that $R \circ S \in \mathrm{WOR}(L)$. Indeed, condition (1) of Definition 1.1 holds trivially. Conditions (2) and (3) are shown by using the same arguments as in the proof of the second lemma in [8], i.e. by using the compatibility of $R$ and the fact that $S$ satisfies condition (2), respectively (3). Now condition (1) yields $\leq \circ R \subseteq R$ and $R \circ \leq \subseteq R$, for any $R \in \mathrm{WOR}(L)$. Since $\leq$ is a reflexive relation, the converse inclusions are clear. Thus we get

$$
\leq \circ R=R \circ \leq=R .
$$

Because $\circ$ is associative, $(\mathrm{U})$ means that $(\operatorname{WOR}(L), \circ)$ is a monoid with unit element $\leq$. (See the same claim and proof in $[8$, p. 2] for ordered relations.)

Now take any $R_{1}, R_{2}, S \in \mathrm{WOR}(L)$, and prove identity (D1). The inclusion $\left(R_{1} \cap R_{2}\right) \circ S \subseteq\left(R_{1} \circ S\right) \cap\left(R_{2} \circ S\right)$ is obvious. In order to prove the converse inclusion, take any $(x, y) \in\left(R_{1} \circ S\right) \cap\left(R_{2} \circ S\right)$. Then there exist some $z_{1}, z_{2} \in L$ such that $\left(x, z_{1}\right) \in R_{1},\left(x, z_{2}\right) \in R_{2}$ and $\left(z_{1}, y\right),\left(z_{2}, y\right) \in S$. In view of condition (1), $z_{1}, z_{2} \leq z_{1} \vee z_{2}$ implies $\left(x, z_{1} \vee z_{2}\right) \in R_{1} \cap R_{2}$, and applying condition (2) for $S$ we get $\left(z_{1} \vee z_{2}, y\right) \in S$. Hence we obtain $(x, y) \in\left(R_{1} \cap R_{2}\right) \circ S$, proving identity (D1). (D2) is proved similarly.

The proof of the first theorem in [8] uses the same argument to show that a stronger form of (D1) and (D2) holds for the ordered relations of a complete lattice. We note also that (D1) and (D2) are valid in any algebra with a (ternary) majority term. As a consequence of Proposition 2.2 we obtain 
Corollary 2.3. Let $R$ be a binary relation on the lattice $L$. Then the following are equivalent:

(i) $R$ is a reflexive weak ordered relation;

(ii) $R$ is a reflexive compatible relation on $L$ which satisfies $\leq \circ R \circ \leq=R$;

(iii) $R$ is a compatible relation containing the order relation $\leq$.

Proof. (i) $\Rightarrow$ (ii). We already know that any weak ordered relation $R$ is compatible. As $\leq$ is the unit of the monoid (WOR $(L), \circ), \leq \circ R \circ \leq=R$ holds. (ii) $\Rightarrow$ (iii). Since $R$ is reflexive, $\leq \circ R \circ \leq=R$ yields $\leq \subseteq R$. (iii) $\Rightarrow$ (i). As $\triangle=\{(x, x) \mid x \in L\}$ is included in $\leq, R$ is reflexive. Because $R$ is compatible, conditions (2) and (3) of Definition 1.1 hold for $R$. Let $u, x, y, v \in L, u \leq x$, $(x, y) \in R$ and $y \leq v$. Then $(u \wedge v, v) \in R$ and $(u, x \wedge y)=(u, x) \wedge(x, y) \in R$. Hence $(u, v)=(u, x \wedge y) \vee(u \wedge v, v) \in R$, thus condition (1) holds also.

Corollary 2.3(iii) implies that $\operatorname{ReWOR}(L)$ is a principal filter in the lattice of all compatible binary relations of $L$. In view of [5, Prop. 2.10], reflexive ordered relations also form a principal filter in the lattice of all completely compatible binary relations of a complete lattice. Denote the set of compatible reflexive relations of $L$ by $\operatorname{Re}(L)$. The fact that $\operatorname{Re}(L)$ forms an algebraic lattice with respect to $\subseteq$ belongs to the folklore, see e.g. [7]. Some other useful properties of these relations are presented below:

Lemma 2.4. Let $L$ be a lattice, $R \in \operatorname{Re}(L)$ and $T \in \operatorname{Tol}(L)$. The line splittings below (in the announcement of Lemma 2.4) are not necessary. Fortunately they do not appear in pdf form Then the following assertions hold true:

(a) $\leq \circ R \circ \leq=\leq \circ R=R \circ \leq$;

(b) $\leq \circ T \in \operatorname{ReWOR}(L)$;

(c) For arbitrary $T_{1}, T_{2} \in \operatorname{Tol}(L)$, we have $T_{1}=T_{2} \Leftrightarrow T_{1} \cap \leq=T_{2} \cap \leq$.

Proof. (a) $\leq \circ R \subseteq \leq \circ R \circ \leq$ is clear. Take any $(a, b) \in \leq \circ R \circ \leq$. Then there exist $c, d \in L$ with $a \leq c,(c, d) \in R$ and $d \leq b$. Now $(b, b) \in R$ yields $(c \vee b, b)=(c \vee b, d \vee b) \in R$. As $a \leq c \vee b$, we get $(a, b) \in \leq \circ R$. Hence $\leq \circ R=\leq \circ R \circ \leq$. $R \circ \leq=\leq \circ R \circ \leq$ is shown similarly. (b) $R:=\leq \circ T=\leq \circ T \circ \leq$ is a reflexive compatible relation and $\leq \circ R \circ \leq=R$, because $\leq \circ \leq=\leq$. Hence, in view of Corollary 2.3(ii), $R \in \operatorname{ReWOR}(L)$. (c) This equivalence belongs to the folklore, see e.g. [2].

The next result was established in [5, Thm. 12], for ordered relations. Since infinite joins and meets and the completeness of the lattice are not used in its proof, this remains valid also in our case, i.e, for reflexive weak ordered relations. We will show only that the maps below are well-defined.

Theorem 2.5. The mappings

$$
\begin{aligned}
& \alpha: \operatorname{ReWOR}(L) \rightarrow \operatorname{Tol}(L), \alpha(R)=R \cap R^{-1} \text { and } \\
& \beta: \operatorname{Tol}(L) \rightarrow \operatorname{ReWOR}(L), \beta(T)=\leq \circ T
\end{aligned}
$$

are lattice isomorphisms and they are inverses of each other.

Proof. Obviously, if $R$ is a compatible reflexive relation on the lattice $L$ then $\alpha(R)=R \cap R^{-1} \in \operatorname{Tol}(L)$. In view of Lemma 2.4(b), for any $T \in \operatorname{Tol}(L)$ we have $\beta(T)=\leq \circ T \in \operatorname{ReWOR}(L)$. Thus $\alpha$ and $\beta$ are correctly defined. 
The following corollary is obvious:

Corollary 2.6. Let $L$ be an arbitrary lattice. Then

(i) $T=(\leq \circ T) \cap(T \circ \geq)$, for every $T \in \operatorname{Tol}(L)$.

(ii) Any reflexive weak ordered relation $R \subseteq L^{2}$ has the form $R=\leq 0$ $\left(R \cap R^{-1}\right)$, i.e. it can be derived from a tolerance $S=R \cap R^{-1}$.

\section{Some notions from Formal Concept Analysis}

A formal context is a triple $\mathcal{K}=(G, M, I)$, where $G$ and $M$ are sets and $I \subseteq$ $G \times M$ is a binary relation. The basic notions of Formal Concept Analysis can be found e.g. in [4] or [9]. By defining for all subsets $A \subseteq G$ and $B \subseteq M$

$$
\begin{aligned}
& A^{I}:=\{m \in M \mid(g, m) \in I, \text { for all } g \in A\}, \\
& { }^{I} B:=\{g \in G \mid(g, m) \in I, \text { for all } m \in B\}
\end{aligned}
$$

we establish a Galois connection between the power-set lattices $\wp(G)$ and $\wp(M)$. We will use the notations ${ }^{I}\left(A^{I}\right)=A^{I I}$ and $\left({ }^{I} B\right)^{I}=B^{I I}$, for any $A \subseteq G$ and $B \subseteq M$. The obtained maps $A \rightarrow A^{I I}, A \subseteq G$ and $B \rightarrow B^{I I}$, $B \subseteq M$ are closure operators on $\wp(G)$ and $\wp(M)$, respectively.

A formal concept of the context $\mathcal{K}$ is a pair $(A, B) \in \wp(G) \times \wp(M)$ with $A^{I}=B$ and ${ }^{I} B=A$, where the set $A$ is called the extent and $B$ is called the intent of the concept $(A, B)$. It is easy to check that a pair $(A, B) \in$ $\wp(G) \times \wp(M)$ is a concept if and only if $(A, B)=\left(A^{I I}, A^{I}\right)=\left({ }^{I} B, B^{I I}\right)$. The concepts of the context $(G, M, I)$ can also be characterized as those pairs $(A, B) \in \wp(G) \times \wp(M)$ whose products $A \times B$ are maximal with respect to the property $A \times B \subseteq I$. The set of all concepts of the context $\mathcal{K}$ is denoted by $\mathcal{L}(\mathcal{K})$. Ordering $\overline{\mathcal{L}}(\mathcal{K})=\mathcal{L}(G, M, I)$ as follows

$$
\left(A_{1}, B_{1}\right) \leq\left(A_{2}, B_{2}\right) \Leftrightarrow A_{1} \subseteq A_{2} \Leftrightarrow B_{1} \supseteq B_{2},
$$

we obtain a complete lattice, the concept lattice of the context $\mathcal{K}=(G, M, I)$. Let us consider the concepts $\gamma(x)=\left(\{x\}^{I I},\{x\}^{I}\right)$ and $\mu(y)=\left({ }^{I}\{y\},\{y\}^{I I}\right)$, for any $x \in G$ and $y \in M$. It can be easily proved that for any concept $(A, B) \in \mathcal{L}(G, M, I)$, we have in $\mathcal{L}(G, M, I)$ :

$$
(A, B)=\bigvee\{\gamma(x) \mid x \in A\}=\bigwedge\{\mu(y) \mid y \in B\} .
$$

The next fact is a part of Basic Theorem on Concept Lattices [4, Thm. 3.]:

Proposition 2.7. A complete lattice $L$ is isomorphic to $\mathcal{L}(G, M, I)$ if and only if there are some mappings $\widetilde{\gamma}: G \rightarrow L$ and $\widetilde{\mu}: M \rightarrow L$ such that the set $\{\widetilde{\gamma}(g) \mid g \in G\}$ is supremum-dense in $L,\{\widetilde{\mu}(m) \mid m \in M\}$ is infimum-dense in $L$ and $(g, m) \in I$ is equivalent to $\widetilde{\gamma}(g) \leq \widetilde{\mu}(m)$, for all $g \in G$ and $m \in M$.

\section{Concept lattices induced by weak ordered relations}

Proposition 3.1. Let $L$ be a lattice, $R \subseteq L^{2}$ a weak ordered relation, and $(A, B)$ a concept of the context $(L, L, R)$. Then $A$ is an ideal and $B$ is a filter in $L$. 
Proof. Suppose that $x \leq a$ for some $a \in A$ and $x \in L$. Since $(a, b) \in R$ for all $a \in A$ and $b \in B$ and $R$ is a weak ordered relation, we obtain $(x, b) \in R$, for all $b \in B$. Hence $x \in{ }^{I} B=A$. Now let $a_{1}, a_{2} \in A$. Then for each $b \in B$ the relations $\left(a_{1}, b\right),\left(a_{2}, b\right) \in R$ imply $\left(a_{1} \vee a_{2}, b\right) \in R$ (see Definition 1.1(2)). Hence $a_{1} \vee a_{2} \in{ }^{I} B=A$. This proves that $A$ is an ideal of $L$. The fact that $B$ is a filter of $L$ is proved dually.

For any subset $X \subseteq L$ of a lattice $L$, let $[X)$ and $(X]$ denote the filter and the ideal generated by $X$, respectively. We will use the following (see also [1, Lemma 3]).

Lemma 3.2 ([3, Lemma 6]). For any convex sublattice $C$ of the lattice $L$ the equality $C=(C] \cap[C)$ holds. Moreover, if $C$ is the intersection of an ideal $I$ and a filter $F$ of $L$, then $I=(C]$ and $F=[C)$.

Proposition 3.3. Let $T$ be a tolerance of the lattice $L$. Then for any block $C$ of $T$ the pair $((C],[C))$ is the unique concept $(A, B) \in \mathcal{L}(L, L, \leq \circ T)$ with $C=A \cap B$. For any concept $(A, B) \in \mathcal{L}(L, L, \leq \circ T), A \cap B$ is a block of $T$ whenever $A \cap B \neq \emptyset$.

Proof. Denote $R:=\leq \circ T$. Then, in view of Corollary 2.6, $T=R \cap R^{-1}$. Let $C$ be a block of $T$. Then $C \times C \subseteq T \subseteq R$, hence $C \subseteq C^{R}$. Clearly, $\left(C^{R R}, C^{R}\right) \in \mathcal{L}(L, L, R)$ and hence $C^{R R} \times C^{\bar{R}} \subseteq R$. Since $C \subseteq C^{R R}$ always holds, we obtain $C \subseteq C^{R R} \cap C^{R}$. We claim that $C=C^{R R} \cap C^{R}$. Indeed, $\left(C^{R R} \cap C^{R}\right) \times\left(C^{R R} \cap C^{R}\right) \subseteq\left(C^{R R} \times C^{R}\right) \cap\left(C^{R} \times C^{R R}\right) \subseteq R \cap R^{-1}=T$, and this means that $C^{R R} \cap C^{R}$ is a preblock of $T$. Since $C$ is block and $C \subseteq C^{R R} \cap C^{R}$, we obtain $C=C^{R R} \cap C^{R}$. Because $R$ is a weak ordered relation and $\left(C^{R R}, C^{R}\right)$ is a concept of the context $(L, L, R)$, the extent $C^{R R}$ is an ideal of $L$ and the intent $C^{R}$ is a filter of $L$, according to Proposition 3.1. As $C$ is a convex sublattice of $L$, by using Lemma 3.2, we obtain $C^{R R}=(C]$, $C^{R}=[C)$ and $C=(C] \cap[C)$. Then $((C],[C)) \in \mathcal{L}(L, L, \leq \circ T)$ also holds, so $C$ is of the required form. Now, assume that $C=A \cap B$ holds for some concept $(A, B) \in \mathcal{L}(L, L, R)$. Since $A$ is an ideal and $B$ is a filter of $L$, in view of Lemma 3.2 we obtain $(A, B)=((C],[C))$. Therefore $((C],[C))$ is the unique concept with the required property.

Finally, take any $(A, B) \in \mathcal{L}(L, L, R)$ such that $D:=A \cap B \neq \emptyset$. Since $A$ is an ideal and $B$ is a filter of $L$ according to Proposition 3.1, we get that $D$ is a convex sublattice of $L$. Then in view of Lemma 3.2, we have $A=(D]$ and $B=[D)$. Since $D \times D \subseteq(A \times B) \cap(B \times A) \subseteq R \cap R^{-1}=T, D$ is a preblock of $T$. Then there exists (at least one) block $C$ of $T$ such that $D \subseteq C$. Then $((C],[C))$ is a concept of the context $(L, L, R)$, moreover, we have $A=(D] \subseteq(C]$ and $B=[D) \subseteq[C)$. Since $(A, B)$ is also a concept of the same context, these relations imply $(A, B)=((C],[C))$. Then $D=A \cap B=(C] \cap[C)=C$. This proves that $A \cap B$ is a block of $T$.

Proposition 3.4. Let $T$ be a tolerance on the lattice $L$. Then the mapping

$$
\delta: L / T \rightarrow \mathcal{L}(L, L, \leq \circ T), \delta(C)=((C],[C)), C \in L / T
$$

is a lattice embedding. 
Proof. In view of Proposition 3.3, for any block $C \in L / T$, we have $((C],[C)) \in$ $\mathcal{L}(L, L, \leq \circ T)$, i.e. the map $\delta$ is well-defined. Assume that $\delta(C)=\delta(D)$ for some $C, D \in L / T$. Then $((C],[C))=((D],[D))$ and Proposition 3.3 imply $C=(C] \cap[C)=(D] \cap[D)=D$, proving that $\delta$ is one to one.

Further, denote $R:=\leq \circ T$, and suppose that $B_{1} \vee B_{2}=E$ holds in the factor lattice $L / T$, for some $B_{1}, B_{2}, E \in L / T$. Now, by [1, Lemma 4] we have:

$$
\left(B_{1} \cup B_{2}\right] \subseteq(E],\left[B_{1}\right) \cap\left[B_{2}\right)=[E) .
$$

Then we get $\delta\left(B_{1}\right)=\left(\left(B_{1}\right],\left[B_{1}\right)\right), \delta\left(B_{2}\right)=\left(\left(B_{2}\right],\left[B_{2}\right)\right)$ and $\delta\left(B_{1} \vee B_{2}\right)=$ $((E],[E))=\left({ }^{R}[E),[E)\right)$. On the other hand, in view of $[4]$ (or [9]), the join operation in $\mathcal{L}(L, L, R)$ has the form

$$
\left(\left(B_{1}\right],\left[B_{1}\right)\right) \vee\left(\left(B_{2}\right],\left[B_{2}\right)\right)=\left({ }^{R}\left(\left[B_{1}\right) \cap\left[B_{2}\right)\right),\left[B_{1}\right) \cap\left[B_{2}\right)\right) .
$$

Since $[E]=\left[B_{1}\right) \cap\left[B_{2}\right)$, we obtain

$$
\delta\left(B_{1} \vee B_{2}\right)=\left({ }^{R}[E),[E)\right)=\left(\left(B_{1}\right],\left[B_{1}\right)\right) \vee\left(\left(B_{2}\right],\left[B_{2}\right)\right)=\delta\left(B_{1}\right) \vee \delta\left(B_{2}\right) .
$$

The equality $\delta\left(B_{1} \wedge B_{2}\right)=\delta\left(B_{1}\right) \wedge \delta\left(B_{2}\right)$ is proved dually. Thus $\delta$ is a lattice embedding.

We note that the embedding $\delta$ is not surjective in general. This is because the concept lattice $\mathcal{L}(L, L, \leq \circ T)$ is always complete, however $L / T$ is not necessarily so, for example, if $L$ is a non-complete lattice and $T$ is its zero tolerance. Denote the Dedekind-MacNeille completion of a lattice $L$ by $\operatorname{DM}(L)$. It is known that $\operatorname{DM}(L)$ is isomorphic to the concept lattice $\mathcal{L}(L, L, \leq)$, see e.g. [4].

Theorem 3.5. Let $T$ be a tolerance of the lattice $L$ and denote $R=\leq \circ T$. Then $\operatorname{DM}(L / T)$ is isomorphic to the concept lattice $\mathcal{L}(L, L, R)$.

Proof. First, observe that for any $x \in L$, we have $(x, x) \in R$ and this implies $x \in\{x\}^{R}$ and $x \in{ }^{R}\{x\}$. As $x \in\{x\}^{R R}$ always holds, we get $\{x\}^{R R} \cap$ $\{x\}^{R} \neq \emptyset$, and in view of Proposition 3.3 this means that the concept $\gamma(x)=$ $\left(\{x\}^{R R},\{x\}^{R}\right) \in \mathcal{L}(L, L, R)$ has the form $\gamma(x)=((C],[C))$, where $C$ is a block of $T$ such that $C=\{x\}^{R R} \cap\{x\}^{R}$. Thus $C$ contains $x$. Similarly, ${ }^{R}\{x\} \cap\{x\}^{R R} \neq \emptyset$ implies that the concept $\mu(x)=\left({ }^{R}\{x\},\{x\}^{R R}\right)$ has the form $\mu(x)=((D],[D))$, where $D$ is a block of $T$ such that $D={ }^{R}\{x\} \cap\{x\}^{R R} \ni x$. Since, in view of $(\mathrm{E})$, the set $\{\gamma(x) \mid x \in L\}$ is supremum-dense and $\{\mu(x) \mid x \in$ $L\}$ is infimum-dense in $\mathcal{L}(L, L, R)$, we obtain that the set $((B],[B)), B \in L / T$ is both supremum- and infimum-dense in $\mathcal{L}(L, L, R)$.

Now, consider the lattice $\mathcal{L}(L / T, L / T, \leq)$. Since $\operatorname{DM}(L / T)$ is isomorphic to $\mathcal{L}(L / T, L / T, \leq)$, to prove our theorem it is enough to show the isomorphism $\mathcal{L}(L / T, L / T, \leq) \cong \mathcal{L}(L, L, R)$. In order to apply Proposition 2.7 , define the maps $\widetilde{\gamma}: L / T \rightarrow \mathcal{L}(L, L, R), \widetilde{\mu}: L / T \rightarrow \mathcal{L}(L, L, R)$ to be equal to the map

$$
\delta: L / T \rightarrow \mathcal{L}(L, L, R), \delta(B)=((B],[B)), B \in L / T,
$$

i.e. let $\widetilde{\gamma}=\widetilde{\mu}=\delta$. Then $\{\widetilde{\gamma}(B) \mid B \in L / T\}=\{((B],[B)) \mid B \in L / T\}$ is supremum-dense and $\{\widetilde{\mu}(B) \mid B \in L / T\}=\{((B],[B)) \mid B \in L / T\}$ is infimumdense in $\mathcal{L}(L, L, R)$. 
Finally, take any $B, C \in L / T$. As in view of Proposition 3.4, $\delta$ is a lattice embedding, we have $B \leq C \Leftrightarrow \widetilde{\gamma}(B)=\delta(B) \leq \delta(C)=\widetilde{\mu}(C)$. Hence, Proposition 2.7 yields $\mathcal{L}(L / T, L / T, \leq) \cong \mathcal{L}(L, L, R)$, completing our proof.

If $L / T$ is a complete lattice, then $\operatorname{DM}(L / T)=L / T$. Hence we obtain:

Corollary 3.6. Let $T$ be a tolerance of the lattice $L$ such that the factor lattice $L / T$ is complete. Then $L / T \cong \mathcal{L}(L, L, \leq \circ T)$.

Remark 3.7. This is the case when the factor lattice $L / T$ is finite. The same result, i.e. $L / T \cong L(L, L, \leq \circ T)$ we obtain also for a complete tolerance $T$ of a complete lattice $L$, because then $L / T$ is a complete lattice. This isomorphism for complete tolerances is also established in [6].

\section{Acknowledgements}

The author thanks the anonymous referees for their valuable remarks on the manuscript.

Funding Open access funding provided by University of Miskolc.

Open Access. This article is licensed under a Creative Commons Attribution 4.0 International License, which permits use, sharing, adaptation, distribution and reproduction in any medium or format, as long as you give appropriate credit to the original author(s) and the source, provide a link to the Creative Commons licence, and indicate if changes were made. The images or other third party material in this article are included in the article's Creative Commons licence, unless indicated otherwise in a credit line to the material. If material is not included in the article's Creative Commons licence and your intended use is not permitted by statutory regulation or exceeds the permitted use, you will need to obtain permission directly from the copyright holder. To view a copy of this licence, visit http://creativecommons. org/licenses/by/4.0/.

Publisher's Note Springer Nature remains neutral with regard to jurisdictional claims in published maps and institutional affiliations.

\section{References}

[1] Czédli, G.: Factor lattices by tolerances. Acta Sci. Math. (Szeged) 44, 35-42 (1982)

[2] Chajda, I., Zelinka, B.: Tolerance relations on lattices. Čas. Pĕst. Mat. 99, 394-399 (1974)

[3] Grätzer, G.: General Lattice Theory. Akademie, Berlin (1978)

[4] Ganter, B., Wille, R.: Formal Concept Analysis: Mathematical Foundations. Springer, Berlin (1999) 
[5] Kaarli, K., Radeleczki, S.: Representation of integral quantales by tolerances. Algebra Universalis 79(1), 1-20 (2018)

[6] Kaarli, K., Radeleczki, S.: Ordered relations, concept lattices and self-bonds. Journal of Multiple-Valued Logic and Soft Computing, to appear in Volume dedicated to the memory of Ivo Rosenberg.

[7] Pöschel, R., Radeleczki, S.: Related structures with involution. Acta Math. Acad. Sci. Hung. 123(1-2), 169-185 (2009)

[8] Valentini, S.: Representation theorems for quantales. Math. Log. Q. 40, 182-190 (1994)

[9] Wille, R.: Restructuring lattice theory: an approach based on hierarchies of concepts. In: Rival, I. (ed.) Ordered Sets, pp. 445-470. Reidel, Dordrecht (1982)

Sándor Radeleczki

Institute of Mathematics

University of Miskolc

Miskolc

Hungary

e-mail: matradi@uni-miskolc.hu

Received: 21 April 2020.

Accepted: 16 January 2021. 